\title{
Pre-hospital use of inhaled corticosteroids and inhaled beta agonists and incidence of ARDS: A population-based study
}

\author{
Asif Muhammad Mangi ${ }^{1}$, Vikas Bansal ${ }^{1}$, Guangxi Li ${ }^{2}$, Matthew S. Pieper ${ }^{2}$, \\ Ognjen Gajic ${ }^{2}$, Emir Festic ${ }^{1^{*}}$
}

${ }^{1}$ Pulmonary and Critical Care Medicine Mayo Clinic, Jacksonville, FL, ${ }^{2}$ Pulmonary and Critical Care Medicine, Mayo Clinic Rochester, MN

\footnotetext{
${ }^{*}$ Corresponding author: festic.emir@mayo.edu Tel.: +19049563331

Fax.: +1 9049532848
}

Received: 10 February 2015

Accepted: 19 July 2015

Key words: ARDS - Corticosteroids • Beta-agonists • Pneumonia
Objective. Inhaled corticosteroids and inhaled beta agonists were shown to decrease the lung injury in animal models. We investigated the association of pre-hospital use of inhaled corticosteroids and inhaled beta agonists with the incidence of Acute Respiratory Distress Syndrome (ARDS) in a population based cohort of hospitalized patients. Material and methods. Retrospective cohort study of adult patients from Olmsted County, Minnesota admitted to the hospital with at least one predisposing condition for ARDS from 2001-2008. The association with pre-hospital use of inhaled corticosteroids and inhaled beta agonists was evaluated using univariate and multivariate analyses. Primary outcome was ARDS and secondary outcome was hospital mortality. Results. Out of 2429 hospitalized adult patients with at least one risk factor for ARDS, 10.5\% of those taking and 14\% of those not taking inhaled corticosteroids developed ARDS (OR 0.72; $0.53-0.97 ; \mathrm{p}<0.03)$. Inhaled beta agonists showed similar unadjusted protective effect; $9.7 \%$ of users and $14.4 \%$ of non-users developed ARDS (OR 0.64; 0.48-0.86; $\mathrm{p}=0.003$ ). After adjusting for risk factors, comorbidities and severity of illness in the multiple logistic regression model, use of inhaled beta agonists, but not inhaled corticosteroids, remained independently associated with decreased risk of ARDS (OR 0.48; 0.31-0.72; $\mathrm{p}<0.001$ versus $0.87 ; 0.57-1.29$; $\mathrm{p}=0.49$ ). The estimated protective effects were more pronounced among patients with pneumonia compared to those without pneumonia. Conclusion. Prehospital use of inhaled beta agonists but not inhaled corticosteroids was significantly associated with decreased incidence of ARDS among hospitalized patients at risk, once adjusted for baseline characteristics, predisposing and comorbid conditions, as well as severity of illness.

\section{Introduction}

Acute respiratory distress syndrome (ARDS) is a heterogeneous syndrome of dysregulated inflammation, resulting from direct insults (e.g. aspiration, pneumonia, chest contusion, etc.), indirect insults (e.g. sepsis, shock, pancreatitis, etc.) or capillarystress failure (e.g. ventilator induced lung injury, high altitude pulmonary edema, etc.) (1). It leads to respiratory failure with a mor- tality of up to $40 \%(2,3)$. Besides attributable morbidity and mortality, ARDS leads to enormous increase in health care cost (4). Although ARDS definition has recently been updated (5), the management remains largely supportive without the therapeutic breakthroughs despite 30 years of intense research in the field. This lead to the recent paradigm shift where the investigative efforts are directed more towards the early 
identification of patients at risk and prevention of ARDS.

Corticosteroids are potent anti-inflammatory agents, however previous studies of systemic corticosteroids were not fully supportive of their protective effect in ARDS patients (6-12). This could be at least in part due to systemic side effects (11). On the contrary, inhaled corticosteroids (ICS) with similar anti-inflammatory properties and direct delivery to the target organ are largely void of systemic adverse effects. Despite heterogeneity in timing of administration or the insult to the lungs, animal studies had shown promising results in attenuation of acute lung injury with pretreatment or concomitant treatments with ICS (13-17). In a large multicenter LIPS cohort we have previously shown that the proposed protective effect of ICS towards ARDS development in patients at risk is more pronounced in patients exposed to the direct, rather than indirect mechanisms of acute lung injury (18). Nearly $70 \%$ of patients on ICS in this cohort were taking inhaled beta agonists (IBA), as well. Beta agonists were previously shown to be able to enhance resolution of pulmonary edema and maintain stability of alveolocapillary membrane under baseline conditions $(19,20)$. We postulated that regular use of ICS and/or IBA could favorably modify dysregulated inflammatory response and result in lesser risk for the development of ARDS Therefore, we hypothesized that patients using ICS and/or IBA prior to hospitalization compared to those who were not using these medications have lower risk of ARDS development after the hospital admission.

In order to test this hypothesis, we studied a population-based cohort of patients at risk for ARDS from Olmsted County, MN.

\section{Material and methods}

The study was approved by Mayo Clinic's institutional review board as a minimal risk study (approval number 08-007804).

\section{Study design}

This was an observational retrospective cohort study designed to test whether the patients at risk of ARDS had smaller risk of developing ARDS if they used ICS and/or IBA prior to admission to the hospital. The cohort was first divided into ICS and IBA users and non-users. Subsequently, we divided the patients based on having admission diagnosis of pneumonia or not.

\section{Study population}

Population included adult patients from Olmsted County, MN, admitted to hospital with at least one risk factor for ARDS, from $2001-2008$. The patients were excluded if they had ARDS already at the time of the admission (first 6 hours), if they died in the emergency room, or restricted their care to the comfort or hospice care.

\section{Predictor variables}

The ICS and IBA therapy exposure was determined through the electronic medical record with the consent of the patient or family. Any ICS or IBA medication either alone or in any combination was included. Baseline characteristics included: age, gender, race and Charlson comorbidity score. Previous diagnoses of chronic obstructive pulmonary disease (COPD) or asthma were included as well, as majority of patients on ICS and/or IBA were expected to have one of these two diagnoses. Clinical variables established as risk factors for ARDS development evident prior to the ARDS diagnosis were: pneumonia, pancreatitis, sepsis, shock, trauma, and multiple transfusions. APACHE III score was used as a severity of illness measure.

\section{Outcome variables}

The ARDS development was the primary outcome during hospitalization. Although 
at the time of study conception the AECC standard criteria were used (21), we adapted the newer Berlin definition for ARDS and excluded patients who did not fulfill the PEEP requirement of at least $5 \mathrm{~cm}$. The secondary outcome was hospital mortality.

\section{Statistical analysis}

We first assessed the distribution of the variables. The continuous variables were reported as medians with $1^{\text {st }}$ and $3^{\text {rd }}$ interquartile ranges (IQR), and categorical variables were reported as counts and proportions. We then performed univariate analyses for the primary and secondary outcomes based on the pre-hospital use of ICS and/or IBA. Subsequently, separate univariate analyses were done on patients with diagnosis of pneumonia and those without it. The statistical test used for univariate analyses was Pearson's Chi-Square test as all expected cell values were greater than 5 . Following this, we performed multivariate logistic regression for primary and secondary outcomes on all patients by including the following variables: ICS and/or IBA, age, Charlson score, abovementioned risk factors for ARDS, asthma and/or COPD and APACHE III score. We evaluated interaction between ICS and IBA in the models. Finally, we applied a multivariate logistic regression model to a subgroup of patients who had pneumonia on admission. The only variable removed from this particulate model was pneumonia. The risk estimates were reported as odds ratios (OR) with 95\% confidence intervals (CI). $\mathrm{P}$ value $<0.05$ was considered statistically significant. JMP 9.0 software and SAS 9.1.3 (SAS Institute Inc., Cary, NC) were used for statistical analysis.

\section{Results}

There were total of 2,429 patients who were admitted to the hospital with at least one risk factor for ARDS during the study period. Median age was 71 years (53-83) and 53\% of patients were male. Other baseline characteristics are listed in Table 1. Of all patients, 564 were taking ICS and 626 were taking IBA with 354 taking both of these medications at the time of admission. There were 996 patients with the diagnosis of pneumonia and 1,433 without. Among 320 patients with ARDS, at the time of diagnosis, there were $158(49 \%)$ with mild, 100 (31\%) with moderate, and 62 (19\%) with severe ARDS, respectively. Median partial pressure of arterial oxygen to fractional inspired oxygen concentration $(\mathrm{P} / \mathrm{F})$ ratios among patients taking and not taking inhaled medications were $106(59 ; 197)$ and $95(54 ; 176)$ for ICS and $128(71 ; 198)$ and $95(53 ; 175)$ for IBA, respectively.

Table 1 Demographic and clinical characteristics of the patients $(n=2,429)$

\begin{tabular}{ll}
\hline Age (year, median IQR) & $71(53-83)$ \\
\hline Male sex (\%) & $1,295(53)$ \\
\hline White race (\%) & $2,156(89)$ \\
\hline Asthma and/or COPD (\%) & $1101(45)$ \\
\hline Charlson score, median (IQR) & $3(1-4)$ \\
\hline APACHE III score, median (IQR) & $52(34-72)$ \\
\hline Inhaled corticosteroid users (\%) & $564(23)$ \\
\hline Inhaled beta agonist users (\%) & $626(26)$ \\
\hline Inhaled corticosteroid \& beta agonist users (\%) & $354(15)$ \\
\hline COPD=Chronic obstructive pulmonary disease. &
\end{tabular}

\section{Univariate analysis}

Pre-hospital ICS use was significantly associated with lower risk of ARDS development; $10.5 \%$ of ICS users and $14 \%$ of nonICS users developed ARDS during the hospitalization (OR 0.72, 0.53-0.97, $\mathrm{p}<0.03$ ). However, when stratified by the diagnosis of pneumonia, it appeared that the protective unadjusted effect in all patients was mostly due to the effect on patients with pneumonia (OR 0.49, 0.33-0.72, $\mathrm{p}<0.001$ ), rather 
than among those without pneumonia (OR $0.93,0.59-1.48, \mathrm{p}=0.77)$. In regards to the secondary outcome - mortality, we observed similar results; pre-hospital ICS use was significantly associated with lower mortality in all patients; $15 \%$ of ICS users and $19 \%$ of non-ICS users died during the hospitalization (OR 0.73, 0.56-0.94, $\mathrm{p}=0.016$ ). When stratified by the diagnosis of pneumonia, the protective unadjusted effect was only significant in patients with pneumonia (OR 0.50, 0.36-0.71, $\mathrm{p}<0.001$ ), where among those without pneumonia there was no significant association with mortality (OR $0.92,0.62$ 1.36, $\mathrm{p}=0.66)$ (Table 2).

Pre-hospital IBA use was significantly associated lower risk of ARDS development; $9.7 \%$ of IBA users and $14.4 \%$ of non-IBA users developed ARDS during the hospitalization (OR 0.64; 0.48-0.86; $\mathrm{p}=0.003$ ). However, when stratified by the diagnosis of pneumonia, the protective unadjusted effect in all patients was more pronounced among patients with pneumonia (OR 0.50; $0.34-0.74 ; \mathrm{p}<0.001)$, rather than among those without pneumonia (OR $0.72 ; 0.46-$ 1.13; $\mathrm{p}=0.15$ ). The similar was observed in regards to the mortality; pre-hospital IBA use was significantly associated with lower mortality in all patients; $12.1 \%$ of IBA users and $20.4 \%$ of non-IBA users died during the hospitalization (OR 0.54; 0.41-0.70; $\mathrm{p}<0.001)$. When stratified by the diagnosis of pneumonia, the protective unadjusted effect was more potent in patients with pneumonia (OR 0.45; 0.31-0.63; $\mathrm{p}<0.001$ ), compared to those without pneumonia (OR 0.54; 0.35-0.82; $\mathrm{p}=0.003$ ) (Table 2).

\section{Multivariate analysis}

The multivariate model included 2197 patients (90\%) who had APACHE III score. When adjusted for pertinent potential confounders in a multivariate logistic regression analysis (Table 3), pre-hospital IBA use was independently protective of ARDS among all patients (OR $0.48,0.31-0.72, \mathrm{p}<0.001)$ but not use of ICS (OR 0.87, 0.57-1.3, p=0.49).

Table 2 Univariate analysis for the primary and secondary outcomes

\begin{tabular}{lllllll}
\hline Characteristics & $\begin{array}{l}\text { Patients with } \\
\text { ARDS }(n=320)\end{array}$ & $\begin{array}{l}\text { Patients without } \\
\text { ARDS }(n=2109)\end{array}$ & -value & $\begin{array}{l}\text { Dead } \\
(n=445)\end{array}$ & $\begin{array}{l}\text { Alive } \\
(n=1984)\end{array}$ & $p$-value \\
\hline Age (median; IQR) & $64(48-78)$ & $72(54-83)$ & $<0.001$ & $78(65-86)$ & $69(50-81)$ & $<0.001$ \\
Male (\%) & $181(56.6)$ & $1114(52.8)$ & 0.21 & $247(55.5)$ & $1048(52.8)$ & 0.3 \\
White (\%) & $270(84.4)$ & $1886(89.4)$ & 0.13 & $373(83.8)$ & $1783(90)$ & $<0.001$ \\
Pancreatitis (\%) & $17(5.3)$ & $61(2.9)$ & 0.02 & $13(2.9)$ & $65(3.3)$ & 0.7 \\
Pneumonia (\%) & $168(52.5)$ & $828(39.3)$ & $<0.001$ & $227(51)$ & $769(38.8)$ & $<0.001$ \\
Sepsis (\%) & $142(44.4)$ & $649(30.8)$ & $<0.001$ & $186(41.8)$ & $605(30.5)$ & $<0.001$ \\
Shock (\%) & $85(16)$ & $303(9.3)$ & $<0.001$ & $144(32.4)$ & $244(12.3)$ & $<0.001$ \\
Asthma/COPD (\%) & $132(41.2)$ & $969(46)$ & 0.11 & $199(44.7)$ & $902(45.5)$ & 0.77 \\
Trauma (\%) & $80(25)$ & $747(35.4)$ & $<0.001$ & $70(15.7)$ & $757(38.2)$ & $<0.001$ \\
Multiple transfusions (\%) & $52(16.2)$ & $136(6.4)$ & $<0.001$ & $60(13.5)$ & $128(6.4)$ & $<0.001$ \\
Charlson Score (median; IQR) & $3(2-4)$ & $3(1-4)$ & 0.015 & $4(2-5)$ & $2(1-4)$ & $<0.001$ \\
APACHE III (median; IQR) & $66(47-91)$ & $51(32-69)$ & $<0.001$ & $77(56-107)$ & $49(30-66)$ & $<0.001$ \\
IBA (\%) & $61(19.1)$ & $565(26.8)$ & 0.003 & $76(17.1)$ & $550(27.7)$ & $<0.001$ \\
ICS (\%) & $59(18.4)$ & $505(24)$ & 0.03 & $84(18.9)$ & $480(24.2)$ & 0.01 \\
\hline
\end{tabular}

$A R D S=A c u t e$ respiratory distress syndrome; $C O P D=C h r o n i c$ obstructive pulmonary disease; IBA=Inhaled beta agonists; ICS=Inhaled corticosteroids. 
Table 3 Multivariate analysis for ARDS

\begin{tabular}{lllllll}
\hline \multirow{2}{*}{$\begin{array}{llllll}\text { Characteristics } \\
\text { All patients* } \\
(\mathrm{n}=2197)\end{array}$} & & $\begin{array}{l}\text { Patients with } \\
\text { pneumonia* }(\mathrm{n}=884)\end{array}$ & $\begin{array}{l}\text { Patients without } \\
\text { pneumonia* }(\mathrm{n}=1313)\end{array}$ \\
\cline { 2 - 7 } OR $(95 \% \mathrm{Cl})$ & $\mathrm{p}$-value & OR $(95 \% \mathrm{Cl})$ & $\mathrm{p}$-value & OR (95\% Cl) & $\mathrm{p}$-value \\
\hline Age & $0.97(0.96-0.98)$ & $<0.001$ & $0.98(0.97-0.99)$ & $<0.001$ & $0.97(0.96-0.98)$ & $<0.001$ \\
Pancreatitis & $1.94(0.96-3.7)$ & 0.06 & $1.85(0.54-5.93)$ & 0.31 & $1.53(0.61-3.56)$ & 0.35 \\
Pneumonia & $2.47(1.83-3.35)$ & $<0.001$ & - & - & - & - \\
Sepsis & $1.96(1.45-2.65)$ & $<0.001$ & $1.89(1.22-2.89)$ & 0.004 & $1.32(0.73-2.34)$ & 0.35 \\
Shock & $1.69(1.21-2.37)$ & 0.002 & $2.67(1.56-4.56)$ & $<0.001$ & $0.98(0.56-1.67)$ & 0.95 \\
Asthma/COPD & $1.12(0.82-1.54)$ & 0.45 & $1.06(0.69-1.62)$ & 0.80 & $1.21(0.75-1.92)$ & 0.43 \\
Trauma & $1.69(1.16-2.44)$ & 0.006 & $2.22(1.26-3.8)$ & 0.006 & $1.11(0.57-2.12)$ & 0.76 \\
Multiple transfusion & $2.49(1.67-3.67)$ & $<0.001$ & $1.81(0.92-3.43)$ & 0.08 & $3.11(1.86-5.09)$ & $<0.001$ \\
Charlson Score & $1.00(0.95-1.06)$ & 0.89 & $0.97(0.89-1.05)$ & 0.47 & $1.03(0.94-1.11)$ & 0.52 \\
APACHE III Score & $1.02(1.01-1.02)$ & $<0.001$ & $1.01(1-1.02)$ & 0.005 & $1.025(1.02-1.03)$ & $<0.001$ \\
IBA & $0.48(0.31-0.72)$ & $<0.001$ & $0.45(0.27-0.74)$ & 0.001 & $0.49(0.22-0.99)$ & 0.05 \\
ICS & $0.87(0.57-1.3)$ & 0.51 & $0.86(0.52-1.41)$ & 0.56 & $0.65(0.28-1.35)$ & 0.26 \\
\hline
\end{tabular}

*All patients who had APACHE III score recorded. COPD=Chronic obstructive pulmonary disease; IBA=Inhaled beta agonists; ICS=Inhaled corticosteroids.

The interaction between IBA and ICS was not statistically significant $(\mathrm{p}=0.22)$. This adjusted protective effect of IBA was slightly more pronounced among 884 patients with pneumonia (OR 0.45; $0.27-0.74 ; \mathrm{p}<0.001$ ), compared to 1313 that did not have pneumonia (OR 0.49; 0.22-0.99; $\mathrm{p}=0.05$ ), however, the interaction with ICS variable in the latter subgroup was significant $(\mathrm{p}=0.016)$.

Table 4 Multivariate analysis for mortality

\begin{tabular}{lllllll}
\hline \multirow{2}{*}{ Characteristics } & $\begin{array}{l}\text { All patients* } \\
(\mathrm{n}=2197)\end{array}$ & & \multicolumn{2}{l}{$\begin{array}{l}\text { Patients with } \\
\text { pneumonia* }(\mathrm{n}=884)\end{array}$} & $\begin{array}{l}\text { Patients without } \\
\text { pneumonia* }(\mathrm{n}=1313)\end{array}$ \\
\cline { 2 - 7 } & OR $(95 \% \mathrm{Cl})$ & $\mathrm{p}$-value & OR $(95 \% \mathrm{Cl})$ & $\mathrm{p}$-value & OR $(95 \% \mathrm{Cl})$ & $\mathrm{p}$-value \\
\hline Age & $1.01(1-1.02)$ & $<0.001$ & $1.02(1.01-1.03)$ & $<0.001$ & $1.01(1-1.02)$ & 0.03 \\
Pancreatitis & $0.82(0.37-1.69)$ & 0.60 & $0.98(0.23-3.5)$ & 0.98 & $0.84(0.29-2.14)$ & 0.72 \\
Pneumonia & $1.6(1.19-2.15)$ & 0.001 & - & - & - & - \\
Sepsis & $1.09(0.82-1.44)$ & 0.53 & $1.24(0.82-1.85)$ & 0.3 & $1.04(0.62-1.76)$ & 0.86 \\
Shock & $2.13(1.56-2.92)$ & $<0.001$ & $1.92(1.11-3.29)$ & 0.02 & $2.18(1.33-3.55)$ & 0.002 \\
Asthma/COPD & $0.9(0.67-1.199)$ & 0.47 & $0.99(0.68-1.47)$ & 0.99 & $0.77(0.49-1.18)$ & 0.23 \\
Trauma & $0.76(0.52-1.09)$ & 0.14 & $0.8(0.42-1.44)$ & 0.47 & $0.83(0.45-1.51)$ & 0.54 \\
Multiple transfusion & $2.39(1.6-3.54)$ & $<0.001$ & $2.54(1.36-4.7)$ & 0.004 & $2.29(1.35-3.84)$ & 0.002 \\
Charlson Score & $1.09(1.03-1.15)$ & 0.001 & $1.07(0.99-1.15)$ & 0.07 & $1.09(1.02-1.19)$ & 0.01 \\
APACHE III Score & $1.03(1.02-1.03)$ & $<0.001$ & $1.02(1.01-1.02)$ & $<0.001$ & $1.03(1.03-1.04)$ & $<0.001$ \\
IBA & $0.42(0.28-0.62)$ & $<0.001$ & $0.44(0.27-0.69)$ & $<0.001$ & $0.44(0.21-0.83)$ & 0.01 \\
ICS & $0.88(0.6-1.27)$ & 0.5 & $0.74(0.46-1.17)$ & 0.2 & $1.13(0.59-2.07)$ & 0.71 \\
\hline
\end{tabular}

*All patients who had APACHE III score recorded; COPD=Chronic obstructive pulmonary disease; IBA=Inhaled beta agonists; ICS=Inhaled corticosteroids. 


\section{Discussion}

In this retrospective cohort study of adult patients from Olmsted County in Minnesota, admitted to the hospital with at least one risk factor for ARDS, the pre-hospital use of IBA was significantly associated with decreased risk of ARDS when adjusted for baseline characteristics, predisposing conditions and severity of illness. Although the overall estimated effect of ICS was in the protective range, this did not reach statistical significance. Overall, adjusted protective effects for ARDS were more pronounced in patients with pneumonia in contrast to those who did not have pneumonia. The interaction between IBA and ICS was only significant in a subgroup of patients without pneumonia. This is the first study to our knowledge that showed protective adjusted effect of IBA on development of ARDS among patients at risk.

The definite role of ICS and IBA in the prevention and early treatment of ARDS has not been established yet, despite a solid body of pre-clinical evidence (6-12). Besides the direct delivery to the target organ, ICS and IBA are void of systemic adverse effects, and this makes them the prime candidates for the lung injury prevention, especially due to direct mechanisms (epithelial injury). Recently, we demonstrated that pre-hospital use of ICS exerts stronger protective effect on patients with direct rather than indirect risk factors for ARDS where $70 \%$ of the patients on ICS were receiving IBA, as well (18). Preclinical studies of ICS delivered prior to pulmonary injury showed significantly reduced pro-inflammatory cytokines and improvements in oxygenation among animals in the intervention group $(16,17)$. The beta agonists have previously established its role in maintaining the stability of the barrier function under baseline conditions (20). This is very important as the early use of IBA and/or ICS in patients with relatively intact epithelium may allow the full effect of the medications, prior to the injury seen in fully established ARDS. Timely administration of inhaled medications may result in their more uniform distribution, thus allowing them to exert their protective effects. On the contrary, in fully established ARDS, inhaled medications cannot reach the target site due to heterogeneous nature of ARDS. Moreover, the affected epithelium is dysregulated and dysfunctional, which reduces the response to medications. Perhaps, this can explain while IBA failed to show the therapeutic effect in ALTA study (22). However, this is speculative and further investigative studies are needed to confirm afore mentioned hypotheses. The ongoing Lung Injury Prevention Study with Budesonide and Beta agonist, formoterol (23) is the first randomized controlled trial of ICS and IBA to prevent pulmonary dysfunction and ARDS, which may shed more lights on the topic.

We have previously studied in the LIPS cohort the role of ICS and IBA on ARDS development and showed that ICS, rather than IBA, could potentially have protective effect, especially in a subgroup of patients with at least one risk factor for the direct lung injury. It is not readily apparent why this study showed different result. Although population-based, this study was done at a single site, compared to 22 sites included in the LIPS cohort. Also, in the LIPS cohort we excluded the patients on ICS who were using systemic corticosteroids (SCS), after observing the higher incidence of ARDS in patients on SCS (7.7\%) (2) compared to those on ICS alone (4.7\%) (18). It is quite possible that some of the patients taking ICS in this single-center cohort were receiving SCS, as well. Also, in LIPS cohort studies, we used propensity score matching to account for the hidden bias and confounding, although by doing this we predisposed the results to the risk of overmatching. 
Other limitations of our study need to be recognized. This was a retrospective observational study and as such it was susceptible to inherent bias and confounding. The study was done at a single academic medical center; however, all patients with ARDS in the Olmsted County, MN are treated in one of the two Mayo Clinic hospitals in Rochester, $\mathrm{MN}$, which makes this population-based study. Notably, generalization of the results may be limited due to less diverse population characteristics in Olmsted County. A fraction of patients $(\sim 10 \%)$ was not included in multivariate analyses due to their lack of APACHE III score. Diagnostic ascertainment of ARDS and pneumonia also needs to be mentioned; however this particular limitation is shared with other studies on this topic. We did not have details on the use of ICS and IBA, such as compliance and duration; however this sort of information bias would be due to non-differential misclassification, which would bias results towards the null hypothesis. We used both Charlson score as well as APACHE III score to adjust for the comorbidities and severity of illness. Furthermore, most common risk factors for ARDS were accounted for. However, there could have been other confounding factors that we did not adjust for in the logistic regression analysis, therefore a potential for hidden bias remains.

\section{Conclusion}

Pre-hospital use of IBA but not ICS was independently associated with decreased incidence of ARDS and mortality once adjusted for common risk factors for ARDS, other comorbidities, and severity of illness in a population-based cohort from Olmsted County, MN. Randomized controlled trials are needed to confirm the proposed protective effects of IBA and ICS on the development of ARDS observed in preclinical and observational studies.

\section{What is already known on this topic}

Pre-clinical studies suggest separate protective roles of inhaled corticosteroids and beta agonists towards development of acute lung injury. A recent secondary analysis of a large multicenter cohort suggested possible protective role of inhaled corticosteroids in patients at risk for acute respiratory distress syndrome development by direct mechanisms, of which most common was pneumonia. Nearly $70 \%$ of patients on inhaled corticosteroids in this study were on inhaled beta agonists, as well.

\section{What this study adds}

Our population-based study demonstrates that both inhaled corticosteroids and beta agonists manifested unadjusted protective effects on development of acute respiratory distress syndrome. After adjusting for risk factors, comorbidities and severity of illness, use of inhaled beta agonists remained independently associated with the protective effect. This protective effect was more pronounced in patients with pneumonia as a predisposing factor in all unadjusted and adjusted analyses.

Author contribution: Conception and design: MAM, VB, GL, MSP, OG, EF; Acquisition, analysis and interpretation of data: MAM, VB, GL, MSP, OG, EF; Drafting the article: MAM, VB, GL, MSP, OG, EF; Revising it critically for important intellectual content: MAM, VB, GL, MSP, OG, EF; Approved final version of the manuscript: MAM, VB, GL, MSP, OG, EF.

Conflicts of interest: The authors declare that they have no conflict of interest.

Funding: Supported in part by grants from the $\mathrm{Na}$ tional Center for Advancing Translational Sciences (grant no. 5KL2TR000136-08 and grant no. CTSA UL1 TR000135), a component of the National Institutes of Health (NIH) and Mayo Foundation. The views expressed in this article do not communicate an official position of the NIH and Mayo Foundation.

\section{References}

1. Thompson BT. Corticosteroids for ARDS. Minerva Anestesiol. 2010;76(6):441-7.

2. Karnatovskaia LV, Lee AS, Gajic O, Festic E. The influence of prehospital systemic corticosteroid use on development of acute respiratory distress syndrome and hospital outcomes. Crit Care Med. 2013;41(7):1679-85.

3. Bosma K, Taneja R, Lewis J. Pharmacotherapy for prevention and treatment of acute respiratory distress syndrome: current and experimental approaches. Drugs. 2010;70(10):1255-82.

4. Rubenfeld GD, Herridge MS. Epidemiology and outcomes of acute lung injury. Chest. 2007;131(2):554-62. 
5. ARDS Definition Task Force, Ranieri VM, Rubenfeld GD, Thompson BT, Ferguson ND, Caldwell E, et al. Acute respiratory distress syndrome: the Berlin Definition. JAMA. 2012;307(23):2526-33.

6. Bone RC, Fisher CJ Jr, Clemmer TP, Slotman GJ, Metz CA. Early methylprednisolone treatment for septic syndrome and the adult respiratory distress syndrome. Chest. 1987;92(6):1032-6.

7. Bernard GR, Luce JM, Sprung CL, Rinaldo JE, Tate RM, Sibbald WJ, et al. High-dose corticosteroids in patients with the adult respiratory distress syndrome. N Engl J Med. 1987;317(25):1565-70.

8. Meduri GU, Headley AS, Golden E, Carson SJ, Umberger RA, Kelso T, et al. Effect of prolonged methylprednisolone therapy in unresolving acute respiratory distress syndrome: a randomized controlled trial. JAMA. 1998;280(2):159-65.

9. Keel JB, Hauser M, Stocker R, Baumann PC, Speich R. Established acute respiratory distress syndrome: benefit of corticosteroid rescue therapy. Respiration. 1998;65(4):258-64.

10. Varpula T, Pettilä V, Rintala E, Takkunen O, Valtonen $\mathrm{V}$. Late steroid therapy in primary acute lung injury. Intensive Care Med. 2000;26(5):52631.

11. Steinberg KP, Hudson LD, Goodman RB, Hough CL, Lanken PN, Hyzy R, et al. Efficacy and safety of corticosteroids for persistent acute respiratory distress syndrome. N Engl J Med. 2006;354(16):167184.

12. Meduri GU, Golden E, Freire AX, Taylor E, Zaman M, Carson SJ, et al. Methylprednisolone infusion in early severe ARDS: results of a randomized controlled trial. Chest. 2007;131(4):954-63.

13. Forsgren PE, Modig JA, Dahlback CM, Axelsson BI. Prophylactic treatment with an aerosolized corticosteroid liposome in a porcine model of early ARDS induced by endotoxaemia. Acta Chir Scand. 1990;156(6-7):423-31.

14. Walther S, Jansson I, Berg S, Lennquist S. Pulmonary granulocyte accumulation is reduced by nebulized corticosteroid in septic pigs. Acta Anaesthesiol Scand. 1992;36(7):651-5.

15. Walther S, Jansson I, Berg S, Olsson Rex L, Lennquist $\mathrm{S}$. Corticosteroid by aerosol in septic pigs-effects on pulmonary function and oxygen transport. Intensive Care Med. 1993;19(3):155-60.

16. Wang J, Zhang L, M. Walther S. Inhaled budesonide in experimental chlorine gas lung injury: influence of time interval between injury and treatment. Intensive Care Med. 2002;28(3):352-7.

17. Jansson AH, Eriksson C, Wang X. Effects of budesonide and $\mathrm{N}$-acetylcysteine on acute lung hyperinflation, inflammation and injury in rats. Vascul Pharmacol. 2005;43(2):101-11.

18. Festic E, Ortiz-Diaz E, Lee A, Li G, Kor DJ, Adebola A, et al. Prehospital use of inhaled steroids and incidence of acute lung injury among patients at risk. J Crit Care. 2013;28(6):985-91.

19. Matthay MA, Folkesson HG, Clerici C. Lung epithelial fluid transport and the resolution of pulmonary edema. Physiol Rev. 2002;82(3):569-600.

20. Spindler V, Waschke J. Beta-adrenergic stimulation contributes to maintenance of endothelial barrier functions under baseline conditions. $\mathrm{Mi}$ crocirculation. 2011;18(2):118-27.

21. Bernard GR, Artigas A, Brigham KL, Carlet J, Falke K, Hudson L, et al. The American-European Consensus Conference on ARDS. Definitions, mechanisms, relevant outcomes, and clinical trial coordination. Am J Respir Crit Care Med. 1994;149(3 Pt 1):818-24.

22. National Heart, Lung, and Blood Institute Acute Respiratory Distress Syndrome (ARDS) Clinical Trials Network, Matthay MA, Brower RG, Carson S, Douglas IS, Eisner M, et al. Randomized, placebo-controlled clinical trial of an aerosolized beta(2)-agonist for treatment of acute lung injury. Am J Respir Crit Care Med. 2011;184(5):561-8.

23. Festic E. NCT01783821 LIPS-B: Lung Injury Prevention Study With Budesonide and Beta. Jacksonville: Mayo Clinic; 2013. 